Archives

$20 \mid 1998$

Miroirs de la Raison d'Etat

\title{
Quelques remarques sur l'adhésion
}

\section{Alain Viala}

\section{(2) OpenEdition}

\section{Journals}

\section{Édition électronique}

URL : http://journals.openedition.org/ccrh/2542

DOI : $10.4000 /$ ccrh. 2542

ISSN : 1760-7906

\section{Éditeur}

Centre de recherches historiques - EHESS

\section{Édition imprimée}

Date de publication : 12 avril 1998

ISSN : 0990-9141

\section{Référence électronique}

Alain Viala, "Quelques remarques sur l'adhésion », Les Cahiers du Centre de Recherches Historiques [En ligne], 20 | 1998, mis en ligne le 20 avril 2009, consulté le 21 avril 2019. URL : http:// journals.openedition.org/ccrh/2542 ; DOI : 10.4000/ccrh.2542

Ce document a été généré automatiquement le 21 avril 2019

Article L.111-1 du Code de la propriété intellectuelle. 


\title{
Quelques remarques sur l'adhésion
}

\author{
Alain Viala
}

1 Partons d'un exemple : lorsqu'en 1617 est promulgué un nouveau « Règlement pour les affaires de librairie » (et il y en aura bien d'autres ensuite...), le préambule dit - je cite de mémoire - que la force des lois ne réside pas tant dans leur rigueur que dans le zèle de ceux qui sont chargés de les faire respecter. Ce texte est doublement remarquable. D'une part, il énonce, sous la forme d'un adage familier des juristes, un fait bien connu des historiens : quand une disposition législative est souvent réitérée et sans cesse aggravée dans les sanctions qu'elle annonce, il y a de fortes probabilités pour que ce soit le signe qu'elle était mal respectée dans la pratique. D'autre part, il montre que les gouvernants d'alors avaient claire conscience des enjeux d'adhésion. Par ce terme, j'entends le crédit et la créance que les membres d'une collectivité accordent à une pratique et à une idée. Or il me semble que très souvent la Raison d'État est envisagée du point de vue de ceux qui ont puissance et intérêt pour en instaurer l'usage ; que corrélativement, on s'intéresse aussi, assez souvent, aux résistances à cette imposition; mais qu'on s'arrête assez peu sur l'attitude de ceux qui sont en position de subir et qui non seulement acceptent, se résignent, mais même y trouvent matière à s'investir, qui adhèrent. Et je voudrais avancer à ce sujet deux remarques, ou si l'on préfère, deux suggestions.

2 La première pourrait résonner comme une invite à la prudence. Le fait que la raison d'État se fonde, dans son principe, sur des intérêts supérieurs, c'est-à-dire sur un intérêt de la collectivité qui dépasse ceux de chacun de ses membres, et même la somme de tous, amène à parler volontiers de transcendance. Mais il y a un risque de glisser alors dans une problématique de la religiosité, de la religiosité religieuse si je puis dire, pour la période qui nous intéresse. Rappelons que sacré signifie avant tout séparé. c'est-à-dire interdit à une majorité des membres de la collectivité, qui ne sont pas initiés, qui sont profanes. Le sacré c'est, en termes modernes, un "domaine réservé ». Je ne suis pas certain qu'au moment des guerres de religion, puis quand s'en liquident les séquelles, les propos qui, plus ou moins métaphoriquement « sacralisent » l'État, renvoient à un sacré authentique, c'est-à-dire alors, chrétien. Ce serait assez étrange dans certains cas : l'État se revendique comme étant «au-dessus des partis» (des Ligues) et quand les partis sont en lutte pour 
définir la religion légitime, prendre la religion comme argument, fût-ce métaphorique, ce serait forcément tomber du côté de l'un ou de l'autre parti, ou en créer un de plus. Mais un vocabulaire du sacré peut être un moyen de susciter l'adhésion. Ceux qui sont du côté des initiés y trouvent leur intérêt, puisqu'ils peuvent croire et faire croire que leur domination répond à des intérêts supérieurs et non à leurs intérêts réels. Ce peut donc être un discours du désintéressent affiché. Mais comme tel, un simulacre.

Or le simulacre a sa puissance propre. Et, du côté de ceux qui subissent, il peut y avoir intérêt à y croire. En acceptant le discours du sacré, ils délèguent de fait, ils chargent les initiés de toutes les responsabilités. Y compris, le cas échéant, de toutes les fautes en cas de malheur.

seconde réflexion, ou remarque, ou suggestion, porte sur les formes de simulacre comme puissance efficace en vue de l'adhésion. Simuler, faire semblant, faire comme si c'était vrai, c'est la logique de la fiction, qui est art de feindre, de faire semblable, semblable au vrai. Donc crédible, digne de crédit, de créance, donc croyable et susceptible d'attirer l'adhésion. Si je relève ainsi des glissements de termes, c'est qu'il me semble que le processus d'adhésion est là : le terme problématique est substitué par un autre, semblable, quasi synonyme, qu'on peut prendre pour argent comptant. Auquel on peut accorder crédit.

5 Pour ne pas laisser tout ceci dans l'abstrait, deux exemples. Naudé qui cite, qui entasse les références et les autorités, donne moins des preuves que des simulacres de preuves. Il procède par similitude pour rendre son propos crédible, efficace. En cela, il est grand rhétoricien.

6 Et, second exemple, si l'on ne se place pas du côté de l'éloquence, mais de celui de la fiction, il me semble que le genre de l'épopée est, à cette époque, significatif. De Chapelain et sa Pucelle aux Saint Louis, Clovis et Charlemagne, il y a une floraison impressionnante, en une génération. Celle où, justement, la raison d'État s'impose. Voltaire a eu beau dire qu'elles étaient mauvaises, il a couronné la série avec sa Henriade. Ce qui n'est pas étonnant de la part de l'historien du Siècle de Louis XIV. Les Français n'ont peut-être pas la tête épique, reste qu'il y a là un corpus important et qui a eu de l'écho en son temps.

Voltaire a vendu la mèche. Cette floraison d'épopées va de pair avec l'entreprise de l' Histoire de France de Mézeray. Dans les deux cas, il s'agit de tracer une généalogie, fictive en fait, des rois qui ont fait la France, et par là, de légitimer les Bourbons. Des auteurs comme Chapelain, ou comme Desmarets de Saint-Sorlin pour son Clovis, étaient proches du pouvoir absolutiste. À côté du discours théorique sur les fondements de la raison d'État, il me semble que ces épopées entrent dans une même démarche pour l'adhésion. L'histoire du royaume est jalonnée de moments de doute sur la légitimité d'une branche devenue dynastie. C'est l'origine de la guerre de Cent Ans. Ce tissu d'Histoire et d'histoires, épique, fabrique un simulacre de continuité jusqu'aux Bourbons y compris. Cette fois, c'est Desmarets qui vend la mèche. Dans sa Défense du poème hérö̈que, il dit bien qu'il entend montrer la continuité de la monarchie depuis Clovis, et une continuité voulue par Dieu. Il sacralise et il fonde en religion. Et il adhère, il y croit, du moins ses textes le disent. On voit son intérêt : pour lui, la monarchie française est la plus grande et il se considère comme le, ou du moins un des plus grands poètes tout court. Dans son épopée ou sa Défense, il ne s'agit pas de discours savant, mais bien de fiction. Donc d'un enjeu d'émotion. Et l'efficacité du simulacre pour fonder l'adhésion, c'est la part d'irrationnel qu'il trouve dans le mouvement affectif, émotif. 
8 Si raison signifie bien ratio, rapport, alors, il est bon de regarder ce qui, dans le rapport à l'État, est un rapport non pas seulement imposé et subi, mais accepté et même désiré ; ce qui fait l'adhésion, et qui donc fait aussi le zèle. Il me semble que cet aspect est présent dans plusieurs communications de cette journée, et qu'il mérite d'être souligné et explicitement désigné comme tel : comme l'un des enjeux majeurs de la réflexion. En effet, si l'adhésion est par essence d'ordre affectif, elle est bien, sans trop forcer le jeu de mots, la « déraison d'État » qui fait la force de la raison d'État. 